\title{
Análisis morfométrico de la apófisis coracoides mediante tomografía computarizada en Chile
}

\section{Morphometric Coracoid Process Analysis by Computed Tomography in Chile}

\author{
Sergio Benavente Cerda ${ }^{1}$ Joaquín Villagra Jara ${ }^{2}$ \\ ${ }^{1}$ Traumatólogo y Ortopedista, Universidad Católica de la Santísima \\ Concepción; Hospital Las Higueras, Talcahuano, Chile \\ 2 Interno de Medicina, Universidad Católica de la Santísima \\ Concepción, Chile \\ Address for correspondence Joaquín Villagra Jara, Interno de \\ Medicina, Universidad Católica de la Santísima Concepción, Chile \\ (e-mail: jorivillaj@gmail.com).
}

Rev Chil Ortop Traumatol 2018;59:105-109.

\section{Resumen \\ Palabras Clave \\ - morfometría \\ - apófisis coracoides \\ - tomografía computarizada}

\section{Abstract \\ Keywords \\ - morphometric measurements \\ - coracoid process \\ - computed tomography}

Objetivo Determinar la morfometría de la apófisis coracoides (Cor) mediante tomografía computarizada de hombro (TC hombro) en población chilena.

Método 162 TC hombro (90 género femenino y 72 masculino), edad promedio 55,9 \pm 20,2 años, fueron analizadas mediante sistema computacional IMPAX.

Resultados Largo total Cor promedio de 41,0 $\pm 2,5 \mathrm{~mm}$. Largo promedio del pilar superior de 20,5 $\pm 2 \mathrm{~mm}$. Altura media pilar superior de 9,2 $\pm 1,2 \mathrm{~mm}$. Ancho promedio pilar superior de 10,3 $\pm 1,5 \mathrm{~mm}$. Altura media de la base de 13,4 $\pm 1,8 \mathrm{~mm}$. Conclusión En nuestra población el pilar superior es más ancho que alto y el largo total de Cor es aproximadamente el doble del largo del pilar superior. Todas las dimensiones de Cor son mayores en el género masculino versus femenino. Al comparar la morfometría de Cor, nuestra población es similar a otros grupos étnicos según lo descrito en la literatura.

Nivel de evidencia III.

Purpose Define the morphometric measurements of the coracoid process (Cor) in Chilean population through the analysis of shoulder computed tomography (shoulder CT)

Method 162 shoulder TC (90 females and 72 males), average age $55.9 \pm 20.2$ years, were analyzed by IMPAX software.

Results The mean total Cor length was of $41.0 \pm 2.5 \mathrm{~mm}$. Average upper pillar length was $20.5 \pm 2 \mathrm{~mm}$. The mean upper pillar height was $9.2 \pm 1.2 \mathrm{~mm}$. The mean upper pillar width was $10.3 \pm 1.5 \mathrm{~mm}$. Average base height was $13.4 \pm 1.8 \mathrm{~mm}$.

Conclusions In our population, upper pillar is wider than high and the total Cor length is approximately twice than upper pillar length. All Cor dimensions are greater in males versus females. The Cor morphometric measurements in our population is similar to other ethnic groups as described in the literature.

Level of evidence III. received

June 30, 2018

accepted

October 1, 2018

published online

January 18, 2019
DOI https://doi.org/

10.1055/s-0038-1675389.

ISSN $0716-4548$.
Copyright $(2018$ by Thieme Revinter

Publicações Ltda, Rio de Janeiro, Brazil
License terms

(c) (i) $\ominus$ (\$) 


\section{Introducción}

La apófisis coracoides (Cor) es una proyección de la escápula sobre la cavidad glenoidea. Compuesta por un pilar inferior vertical que se origina en el aspecto anterosuperior de la bóveda glenoidea, unido a un pilar superior horizontal, cada uno con una orientación tridimensional diferente. ${ }^{1,2}$ Corresponde a un importante hito anatómico y quirúrgico, al ser sitio de inserción de estabilizadores estáticos y dinámicos, ${ }^{3,4}$ y punto de referencia para una gran variedad de procedimientos sobre la articulación del hombro. ${ }^{5-10}$

\section{Objetivo}

Determinar la morfometría de Cor mediante tomografía computarizada de hombro (TC hombro) en población chilena.

\section{Metodología}

Diseño de estudio: Estudio descriptivo transversal. Pacientes: Total de pacientes mayores de 18 años, sometidos a TC hombro en un mismo centro, durante los años 2014 a 2017, ingresados en el sistema computacional IMPAX versión 6.3.1.8000 del proveedor AFGA Healthcare, realizados en un equipo marca Toshiba modelo Aquilion 64, con adquisición de imágenes de $1 \mathrm{~mm}$ cada $0,8 \mathrm{~mm}$ con reconstrucción ósea en los planos axial, sagital y coronal de $3 \mathrm{~mm}$ cada $3 \mathrm{~mm}$. Excluyendo aquellos pacientes con uno o más de los siguientes:

Criterios de exclusión:

(a) Antecedente de fractura o cualquier traumatismo de Cor

(b) Antecedente de alguna intervención quirúrgica sobre Cor

(c) Antecedentes o diagnóstico de: lesión acromioclavicular, masas tumorales, luxaciones glenohumerales, fracturas de húmero proximal y/o escápula, que no permitan analizar Cor.

\section{Protocolo de TC Hombro}

Paciente en decúbito supino con la cabeza primero, el brazo contralateral sobre la cabeza y el brazo de estudio apegado al tórax con la mano en supinación. El hombro a estudiar se ubica en el centro del gantry. Se realiza centraje en la articulación glenohumeral. Con scout de 20 a $25 \mathrm{~cm}$, incluyendo toda la escápula y húmero proximal. Se utilizan $120 \mathrm{Kv}, 180$ a 240 $\mathrm{mAs}$, rotación del tubo $\leq 1$ segundo, colimación 0,625 o la menor posible, Pitch $<1$, reconstrucciones MPR de 0,625 con algoritmo óseo y presentación de imágenes en los 3 planos de $3 \mathrm{~mm}$ cada $3 \mathrm{~mm}$.

Plano Sagital: en coronal paralelo al eje del húmero y en axial paralelo a la articulación glenohumeral.

Plano Coronal: en axial perpendicular a la articulación glenohumeral y en sagital paralelo al eje del húmero.

Plano Axial: en coronal perpendicular a la articulación glenohumeral y en sagital perpendicular al eje del húmero.

\section{Mediciones}

Con las herramientas del programa IMPAX se aumentó el nivel de contraste, amplió la imagen y se dividió la pantalla

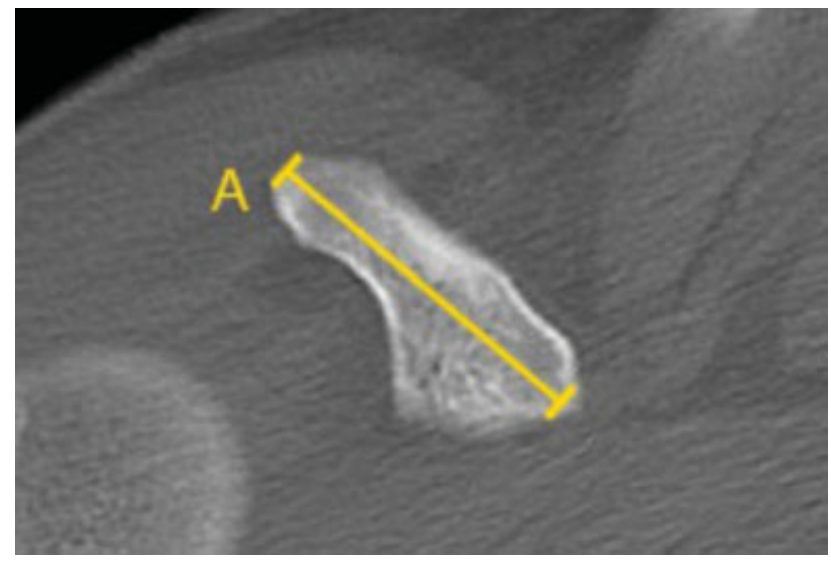

Fig. 1 TC hombro, en plano axial de Cor. (A) Largo total.

para analizar un mismo punto anatómico simultáneamente en las proyecciones axial, sagital y coronal.

Largo total: Distancia desde el inicio del pilar inferior o base, hasta la punta del pilar superior de Cor, medida en plano axial. (-Fig. 1)

Altura del pilar superior: Distancia de mayor longitud de superior a inferior del pilar superior de Cor, medida en plano sagital. (-Fig. 2)

Ancho del pilar superior: Distancia de mayor longitud de lateral a medial del pilar superior de Cor, medida en plano axial. (-Fig. 3 )

Largo del pilar superior: Distancia desde la rodilla (punto específico en el que la propagación de la superficie inferior cambia de una dirección vertical a una horizontal), hasta la punta del pilar superior de Cor, en el plano axial y sagital. (-Figs. 2 y 3 )

Altura de la base: Distancia desde el inicio del pilar inferior o base, tras observar el tubérculo supraglenoídeo, hasta la rodilla de Cor, en un plano sagital. (-Fig. 4)

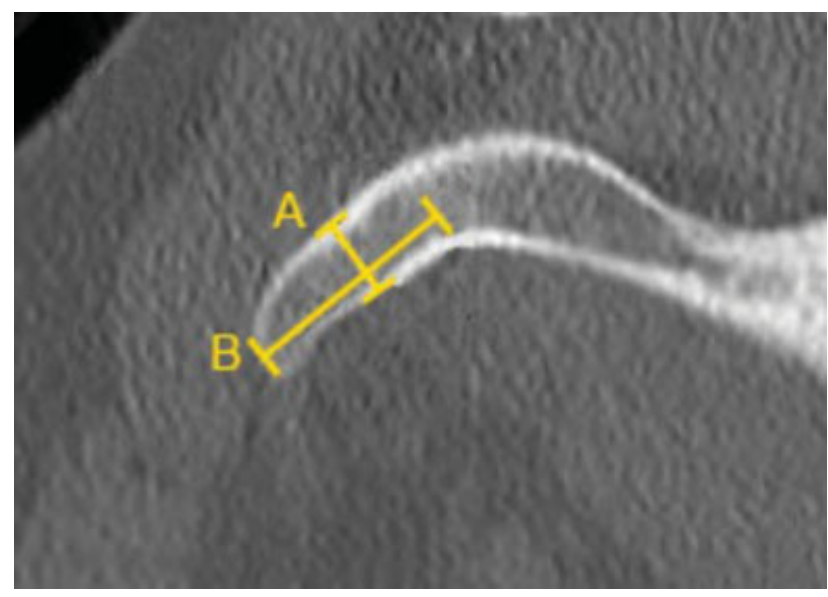

Fig. 2 TC hombro, plano sagital de Cor. Las flechas indican la rodilla de Cor donde termina la base e inicia el pilar superior. (A) Altura del pilar superior, (B) Largo del pilar superior. 


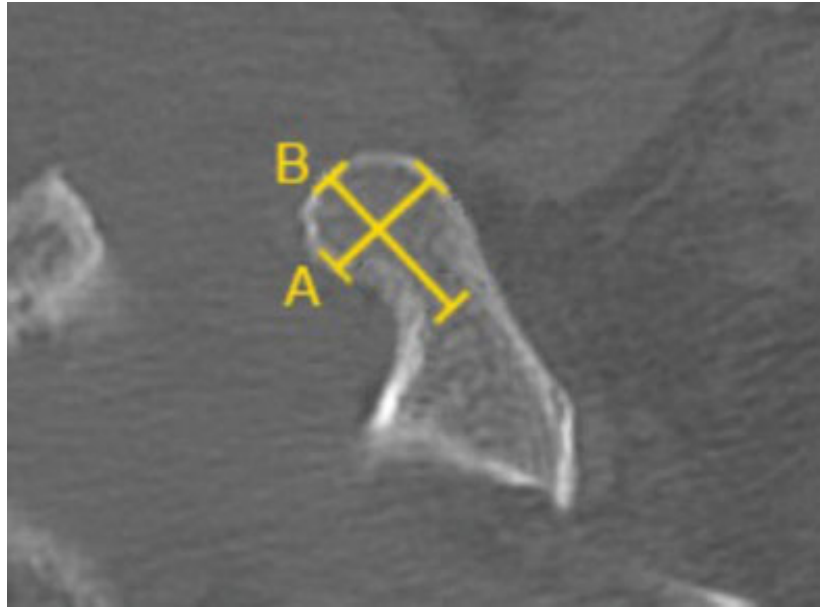

Fig. 3 TC hombro, plano axial de Cor. Las flechas indican la rodilla de Cor donde termina la base e inicia la punta. (A) Ancho del pilar superior, (B) Largo del pilar superior.

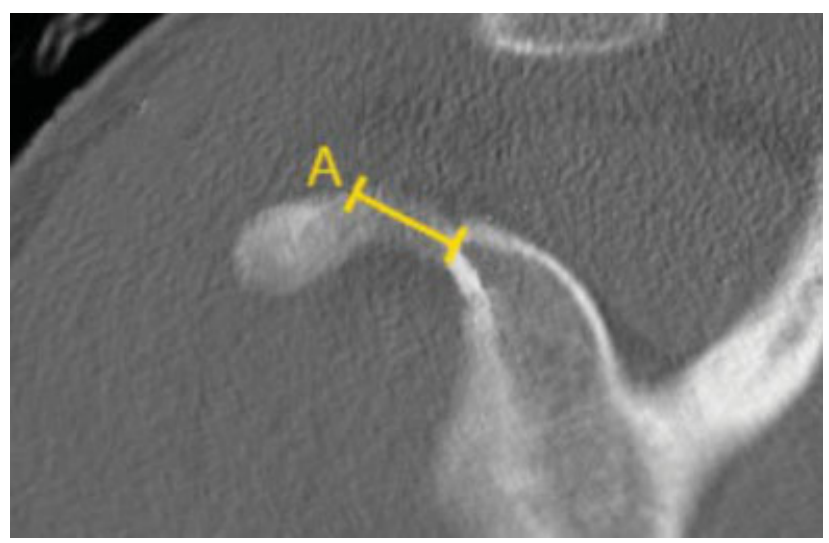

Fig. 4 TC hombro, plano sagital de Cor. Las flechas indican la rodilla de Cor donde termina la base e inicia el pilar superior. (A) Altura de la base.

\section{Resultados}

Se analizaron 162 TC hombro. Distribución por géneros: 90 pacientes sexo femenino y 72 sexo masculino. Lateralidad: 90 derechos y 72 izquierdos. Edad promedio $55,9 \pm 20,2$ años (-Tabla 1).

La morfometría promedio de Cor en la población estudiada se muestra en la $\boldsymbol{- T}$ Tabla 2.

Tabla 1 Descripción de la muestra

\begin{tabular}{|l|l|l|l|}
\hline & $\begin{array}{l}\text { Total } \\
(\boldsymbol{n}=\mathbf{1 6 2})\end{array}$ & $\begin{array}{l}\text { Hombres } \\
(\boldsymbol{n}=\mathbf{7 2})\end{array}$ & $\begin{array}{l}\text { Mujeres } \\
(\boldsymbol{n}=\mathbf{9 0})\end{array}$ \\
\hline Edad $^{\mathrm{a}}$ & $55,9 \pm 20,2$ & $48,3 \pm 19,2$ & $62 \pm 19$ \\
\hline $\begin{array}{l}\text { Escápulas } \\
\text { Derechas }\end{array}$ & 90 & 40 & 50 \\
\hline $\begin{array}{l}\text { Escápulas } \\
\text { izquierdas }\end{array}$ & 72 & 32 & 40 \\
\hline \multicolumn{2}{|l|}{${ }^{\text {aEdad en años }} \pm$ Desviación estándar } \\
\hline
\end{tabular}

\section{Discusión}

La anatomía de Cor es compleja, la base o pilar inferior (porción vertical) se origina en el aspecto anterosuperior de la bóveda glenoidea, está conectado al pilar superior (porción horizontal) en la rodilla (punto específico en el que la propagación de la superficie inferior cambia de una dirección vertical a una horizontal). Luego el pilar superior atraviesa lateralmente y termina en la punta. ${ }^{1,2}$

Existen múltiples estudios sobre la anatomía de Cor, siendo esta la primera vez que se reporta en población chilena (-Tabla 2).

En cuanto a la morfometría según género, nuestros resultados son similares a la literatura, ${ }^{11-20}$ donde todas las dimensiones estudiadas son mayores en género masculino que en femenino, independiente de la etnia y rango de edad de la muestra.

$\mathrm{Al}$ analizar los resultados según etnia, el largo total Cor es la medida más representada, nuestro estudio reporta un promedio de $41,0 \pm 2,5 \mathrm{~mm}$, resultados similares a lo descrito en poblaciones caucásicas, ${ }^{11,14}$ surafricanas ${ }^{12} \mathrm{y}$ asiáticas. ${ }^{18,19}$ Valores mayores, se han presentado en series $^{13,15}$ que no respetan una proporción homogénea entre géneros, estudiando principalmente a hombres. En esos casos, al comparar los subgrupos de género femenino con nuestros resultados, se presentan valores similares. ${ }^{13,15}$ Dugarte y col. ${ }^{20}$, en su serie de 418 especímenes, divididos en 2 grupos según edad, demostró que existe una diferencia estadísticamente significativa en la altura del pilar superior, aumentando su tamaño a medida que aumenta la edad, a expensas de cambios degenerativos osteofíticos de Cor. Eso explica las diferencias en esa medición de nuestra serie (edad promedio de 55,9 años) versus lo reportado por Dolan y col. $^{15}$, cuya muestra de estudio presentaba una edad promedio de 83,8 años. En ese mismo punto, al analizar series con muestras de edad similares a las nuestras, se reportan valores afines en poblaciones caucásicas, ${ }^{11,16}$ caucásica y afroamericanas, ${ }^{13}$ surafricanas $^{12}$ y asiáticas. ${ }^{19}$ Lo mismo ocurrió con las dimensiones del ancho del pilar superior, ${ }^{12,14,16,17,19}$ largo del pilar superior ${ }^{12,14,16,19} \mathrm{y}$ altura de la base. ${ }^{14}$

Los resultados de nuestro estudio sobre la morfometría de Cor, mostraron que el pilar superior es más ancho $(10,3 \pm 1,5 \mathrm{~mm})$ que alto $(9,2 \pm 1,2 \mathrm{~mm})$, y que el largo total $(41,0 \pm 2,5 \mathrm{~mm})$ es aproximadamente el doble que el largo del pilar superior $(20,5 \pm 2 \mathrm{~mm})$. Consideraciones anatómicas que pudiesen ser importantes al momento de elegir la orientación de Cor para la reconstrucción quirúrgica de defectos óseos glenoideos, u otros procedimientos que impliquen un conocimiento de la morfología de Cor.

Consideramos que las ventajas del estudio morfométrico mediante TC, son la excelente definición de contornos óseos, exactitud de herramientas de medición asistidas por software, y la independencia de la calidad del espécimen estudiado y su técnica de disección, evitando además el error en las mediciones secundario a interposición de partes blandas descrita en estudios anatómicos. Cor presenta distintos 
Tabla 2 Morfometría de la apófisis coracoides en población chilena y la literatura

\begin{tabular}{|c|c|c|c|c|c|c|c|c|}
\hline Autores & $\mathrm{N}$ & Género & Etnia & $\begin{array}{l}\text { Largo total } \\
\text { (mm } \pm \mathrm{DS} \text { ) }\end{array}$ & $\begin{array}{l}\text { Altura del } \\
\text { pilar superior } \\
\text { (mm } \pm \mathrm{DS} \text { ) }\end{array}$ & $\begin{array}{l}\text { Ancho del } \\
\text { pilar superior } \\
\text { (mm } \pm \mathrm{DS} \text { ) }\end{array}$ & $\begin{array}{l}\text { Largo del } \\
\text { pilar superior } \\
\text { (mm } \pm \mathrm{DS} \text { ) }\end{array}$ & $\begin{array}{l}\text { Altura de } \\
\text { la base } \\
(\mathrm{mm} \pm \mathrm{DS})\end{array}$ \\
\hline \multirow{3}{*}{$\begin{array}{l}\text { Benavente \& } \\
\text { Villagra } \\
(2018)\end{array}$} & 162 & Ambos & \multirow{3}{*}{$\begin{array}{l}\text { Latino } \\
\text { americanos }\end{array}$} & $41,0 \pm 2,5$ & $9,2 \pm 1,2$ & $10,3 \pm 1,5$ & $20,5 \pm 2$ & $13,4 \pm 1,8$ \\
\hline & 72 & Hombres & & $42,1 \pm 2,5$ & $9,9 \pm 1,2$ & $11,0 \pm 1,2$ & $21,3 \pm 2,1$ & $13,9 \pm 1,7$ \\
\hline & 90 & Mujeres & & $40,1 \pm 2,0$ & $8,7 \pm 0,8$ & $9,8 \pm 1,3$ & $19,8 \pm 1,7$ & $13 \pm 1,8$ \\
\hline $\begin{array}{l}\text { Gumina y col. } \\
\text { (1999) }\end{array}$ & 204 & Ambos & Caucásicos & $38,15 \pm 3,97$ & $7,19 \pm 1,04$ & & & \\
\hline $\begin{array}{l}\text { Bhatia y col. } \\
\text { (2007) }\end{array}$ & 101 & Ambos & Africana & $41,7 \pm 4,7$ & $8,4 \pm 1,5$ & $14,2 \pm 1,9$ & $19,0 \pm 3,3$ & \\
\hline \multirow{3}{*}{$\begin{array}{l}\text { Rios y col. } \\
(2007)\end{array}$} & 120 & Ambos & \multirow{3}{*}{$\begin{array}{l}\text { Caucásicos y } \\
\text { afroamericanos }\end{array}$} & $45,2 \pm 4,1$ & $11,9 \pm 1,8$ & & & \\
\hline & 96 & Hombres & & $46,3 \pm 3,3$ & $12,2 \pm 1,7$ & & & \\
\hline & 24 & Mujeres & & $40,7 \pm 4,3$ & $10,5 \pm 1,6$ & & & \\
\hline \multirow{3}{*}{$\begin{array}{l}\text { Salznmann y col. } \\
\text { (2008) }\end{array}$} & 23 & Ambos & \multirow[t]{3}{*}{ Caucásicos } & $43,1 \pm 2,2$ & $8,2 \pm 1$ & $13,6 \pm 2,1$ & $20,3 \pm 2,6$ & $14,9 \pm 2,4$ \\
\hline & 6 & Hombres & & $46 \pm 1,9$ & $9,6 \pm 0,9$ & $15,2 \pm 1,5$ & $22,9 \pm 1,1$ & $15,4 \pm 1,3$ \\
\hline & 17 & Mujeres & & $42 \pm 1,4$ & $7,8 \pm 0,9$ & $13,1 \pm 2,2$ & $20 \pm 2,1$ & $13,6 \pm 1,7$ \\
\hline $\begin{array}{l}\text { Dolan y col. } \\
\text { (2011) }\end{array}$ & 20 & Ambos & Caucásicos & $45,6 \pm 4,2$ & $11,5 \pm 0,9$ & $18,3 \pm 1,8$ & & \\
\hline $\begin{array}{l}\text { Armitage y col. } \\
\text { (2011) }\end{array}$ & 34 & Ambos & Caucásicos & & $10,5 \pm 1,7$ & $15,0 \pm 2,2$ & $16,8 \pm 2,5$ & \\
\hline $\begin{array}{l}\text { Bueno y col. } \\
\text { (2012) }\end{array}$ & 61 & Ambos & $\begin{array}{l}\text { Latino } \\
\text { americanos }\end{array}$ & & $8.37 \pm 0.93$ & $14,51 \pm 1,9$ & & \\
\hline $\begin{array}{l}\text { Kavita y col. } \\
(2013)\end{array}$ & 129 & Ambos & Asiáticos & $41 \pm 3,9$ & & $7,4 \pm 1,1$ & & \\
\hline $\begin{array}{l}\text { Lian y col. } \\
\text { (2016) }\end{array}$ & 30 & Hombres & Asiáticos & $42,1 \pm 2,31$ & $9,1 \pm 1,75$ & $13,61 \pm 2$ & $24,75 \pm 7,23$ & \\
\hline \multirow[t]{2}{*}{$\begin{array}{l}\text { Dugarte y col. } \\
\text { (2017) }\end{array}$} & 214 & $\begin{array}{l}\text { Ambos } \\
\text { (25-35 años) }\end{array}$ & \multirow[t]{2}{*}{ Caucásicos } & & $\begin{array}{l}\text { M: } 7.86 \\
\text { H: } 9.41\end{array}$ & & & \\
\hline & 204 & $\begin{array}{l}\text { Ambos } \\
(>55 \text { años) }\end{array}$ & & & $\begin{array}{l}\text { M: } 8.08 \\
H: 9.67\end{array}$ & & & \\
\hline
\end{tabular}

grados de angulación ya sea hacia caudal o lateral, confiriéndole una estructura general curvada en forma de gancho o más bien plana. ${ }^{20}$ La principal limitación de este estudio, es la orientación de los cortes de TC, ya que dependiendo de la estructura general de Cor y sus distintos grados de angulación, se pudiesen alterar las mediciones descritas y su análisis.

\section{Conclusión}

En nuestra población, el pilar superior es más ancho que alto y el largo total de Cor es aproximadamente el doble del largo del pilar superior. Todas las dimensiones de Cor son mayores en el género masculino versus femenino. Al comparar la morfometría de Cor, nuestra población es similar a otros grupos étnicos según lo descrito en la literatura.

\section{Conflicto de Interés}

Los autores declaran no presentar conflicto de interés.

Este estudio cuenta con la aprobación del comité ético científico de nuestro servicio.

\section{Bibliografía}

1 Williams PL, Bannister LH, Berry MM, et al. Skeletal system: Scapula. In: Williams PL, Bannister LH, Berry MM, Collins P, Dyson M, Dussek JE, Ferguson MWJ, editors. Gray's Anatomy. 38th Ed. Edinburgh: Churchill Livingston; 1995:615-619

2 Anetzberger H, Putz R. The scapula: principles of construction and stress. Acta Anat (Basel) 1996;156(01):70-80

3 Fukuda K, Craig EV, An KN, Cofield RH, Chao EY. Biomechanical study of the ligamentous system of the acromioclavicular joint. J Bone Joint Surg Am 1986;68(03):434-440

4 Debski RE, Parsons IM IV, Woo SL, Fu FH. Effect of capsular injury on acromioclavicular joint mechanics. J Bone Joint Surg Am 2001; 83-A(09):1344-1351

5 Froimson AI. Fracture of the coracoid process of the scapula. J Bone Joint Surg Am 1978;60(05):710-711

6 Eyres KS, Brooks A, Stanley D. Fractures of the coracoid process. J Bone Joint Surg Br 1995;77(03):425-428

7 Ogawa K, Yoshida A, Takahashi M, Ui M. Fractures of the coracoid process. J Bone Joint Surg Br 1997;79(01):17-19

8 von Schroeder HP, Kuiper SD, Botte MJ. Osseous anatomy of the scapula. Clin Orthop Relat Res 2001;(383):131-139

9 Butters KP. The scapula. In: Rockwood CAJr, Matsen F III, editors. The Shoulder. 2nd ed. Philadelphia, PA: WB Saunders; 1998:391-427

10 Gerber C, Terrier F, Ganz R. The role of the coracoid process in the chronic impingement syndrome. J Bone Joint Surg Br 1985;67 (05):703-708 
11 Gumina S, Postacchini F, Orsina L, Cinotti G. The morphometry of the coracoid process - its aetiologic role in subcoracoid impingement syndrome. Int Orthop 1999;23(04):198-201

12 Bhatia DN, de Beer JF, du Toit DF. Coracoid process anatomy: implications in radiographic imaging and surgery. Clin Anat 2007; 20(07):774-784

13 Rios CG, Arciero RA, Mazzocca AD. Anatomy of the clavicle and coracoid process for reconstruction of the coracoclavicular ligaments. Am J Sports Med 2007;35(05):811-817

14 Salzmann GM, Paul J, Sandmann GH, Imhoff AB, Schöttle PB. The coracoidal insertion of the coracoclavicular ligaments: an anatomic study. Am J Sports Med 2008;36(12):2392-2397

15 Dolan CM, Hariri S, Hart ND, McAdams TR. An anatomic study of the coracoid process as it relates to bone transfer procedures. J Shoulder Elbow Surg 2011;20(03):497-501

16 Armitage MS, Elkinson I, Giles JW, Athwal GS. An anatomic, computed tomographic assessment of the coracoid process with special reference to the congruent-arc latarjet procedure. Arthroscopy 2011;27(11):1485-1489

17 Bueno RS, Ikemoto RY, Nascimento LG, Almeida LH, Strose E, Murachovsky J. Correlation of coracoid thickness and glenoid width: an anatomic morphometric analysis. Am J Sports Med 2012;40(07):1664-1667

18 Kavita P, Singh J. Morphology of coracoid process and glenoid cavity in adult human scapulae. International Journal of Analytical. Pharmaceutical and Biomedical Sciences 2013;2 (02):62-65

19 Lian J, Dong L, Zhao Y, Sun J, Zhang W, Gao C. Anatomical study of the coracoid process in Mongolian male cadavers using the Latarjet procedure. J Orthop Surg Res 2016;11(01):126

20 Dugarte AJ, Davis RJ, Lynch TS, Schickendantz MS, Farrow LD. Anatomic Study of Subcoracoid Morphology in 418 Shoulders: Potential Implications for Subcoracoid Impingement. Orthop J Sports Med 2017;5(10):2325967117731996 